\title{
A Count Invariant for Lambek Calculus with Additives and Bracket Modalities ${ }^{\star}$
}

\author{
Oriol Valentín ${ }^{1}$, Daniel Serret ${ }^{2}$, and Glyn Morrill ${ }^{1}$ \\ 1 Universitat Politècnica de Catalunya \\ oriol.valentin@gmail.com, morrill@lsi.upc.edu \\ http://www.lsi.upc.edu/ morrill/ \\ 2 Universitat de Barcelona \\ daniel.serret@gmail.com
}

\begin{abstract}
The count invariance of van Benthem (1991[16]) is that for a sequent to be a theorem of the Lambek calculus, for each atom, the number of positive occurrences equals the number of negative occurrences. (The same is true for multiplicative linear logic.) The count invariance provides for extensive pruning of the sequent proof search space. In this paper we generalize count invariance to categorial grammar (or linear logic) with additives and bracket modalities. We define by mutual recursion two counts, minimum count and maximum count, and we prove that if a multiplicative-additive sequent is a theorem, then for every atom, the minimum count is less than or equal to zero and the maximum count is greater than or equal to zero; in the case of a purely multiplicative sequent, minimum count and maximum count coincide in such a way as to together reconstitute the van Benthem count criterion. We then define in the same way a bracket count providing a count check for bracket modalities. This allows for efficient pruning of the sequent proof search space in parsing categorial grammar with additives and bracket modalities.
\end{abstract}

\section{Introduction}

Van Benthem (1991[16]) showed that a necessary condition for a sequent to be a theorem of the Lambek calculus is that it satisfies a simple count check. Let $\mathcal{P}$ be the set of atoms. Where $P \in \mathcal{P}$, the $P$-count $\#_{P}(A)$ of a type $A$ is defined by:

$$
\begin{aligned}
& \#_{P}(P)=1 \\
& \#_{P}(Q)=0 \text { for } Q \in \mathcal{P}-\{P\} \\
& \#_{P}(A \bullet B)=\#_{P}(A)+\#_{P}(B) \\
& \#_{P}(A \backslash C)=\#_{P}(C)-\#_{P}(A) \\
& \#_{P}(C / B)=\#_{P}(C)-\#_{P}(B)
\end{aligned}
$$

\footnotetext{
* Research partially supported by an ICREA Acadèmia 2012 to the third author, and by BASMATI MICINN project (TIN2011-27479-C04-03) and SGR2009-1428 (LARCA). Many thanks to Josefina Sierra and to three Formal Grammar referees for comments and suggestions. Particular thanks to the referee who pointed towards the simplification of the proposal in the appendix which we have used in the main text. Any errors are our own.
} 
Let the $P$-count be extended to configurations by the following, where $\Lambda$ is the empty configuration:

$$
\begin{aligned}
& \#_{P}(A, \Gamma)=\#_{P}(A)+\#_{P}(\Gamma) \\
& \#_{P}(\Lambda)=0
\end{aligned}
$$

The count-invariance property is that

$$
\vdash \Gamma \Rightarrow A \Longrightarrow \forall P \in \mathcal{P}, \#_{P}(\Gamma)=\#_{P}(A)
$$

This is proved by induction on sequent proofs.

The count invariance forms the basis of an extensive pruning of the sequent proof search space in categorial parsing-as-deduction/theorem-proving. Every time a subgoal $\Gamma \Rightarrow A$ is generated it can be quickly checked whether $\forall P, \#_{P}(\Gamma)=\#_{P}(A)$; if not, the subgoal can be discarded at once. Informal experimentation shows that such countchecking, together with focusing normalization (Andreoli 1992[1]; König 1989[6]; Hepple 1990[4]; Hendriks 1993[3]; Morrill 2011[11]) makes a critical difference in efficiency of Lambek calculus sequent proof search parsing-as-deduction.

In this paper we consider the case of the Lambek calculus extended with additives (Lambek 1961[7]; Girard 1987[2]; van Benthem 1991[16]; Morrill 1990[9]; Kanazawa 1992[5]). For linguistic motivation see Morrill (1994[14], ch. 6) or Morrill (2011[15], ch. 7). Our contribution is to define two new counts, $\#_{\min }$ and $\#_{\max }$, which, for a multiplicative-additive sequent to be a theorem, must be less than or equal to zero and greater than or equal to zero respectively. In the special case that a sequent has no additives, $\#_{\min }=\#_{\max }=$ the van Benthem count \# so that the two inequations together impose the van Benthem criterion, i.e. our generalisation preserves the particular case of the van Benthem count for pure multiplicative sequents. We go on to formulate in addition count invariance for bracket modalities (Morrill 1992[10]; Moortgat 1995[8]).

\section{Count invariance for additives}

Let us consider the sequent formulation of LA, the Lambek calculus with additive connectives. We will denote the additive conjunction as $\wedge$ and the additive disjunction as $\vee$. The sequent rules are shown in Figure 1. Cut, of course, is eliminable.

Where $m=\max$ or min, we define recursively counts $\#_{\max / \min , P}$ on types as follows: ${ }^{1}$

$$
\begin{aligned}
& \#_{m}(P)=1 \\
& \#_{m}(Q)=0 \text { for } Q \in \mathcal{P}-\{P\} \\
& \#_{m}(A \bullet B)=\#_{m}(A)+\#_{m}(B) \\
& \#_{m}(A \backslash C)=\#_{m}(C)-\#_{m}(A) \\
& \#_{m}(C / B)=\#_{m}(C)-\#_{m}(B) \\
& \#_{m}(A \wedge B)=\bar{m}\left(\#_{m}(A), \#_{m}(B)\right) \\
& \#_{m}(A \vee B)=m\left(\#_{m}(A), \#_{m}(B)\right)
\end{aligned}
$$

We extend the counts $\#_{\min / \max }$ to configurations by the following:

$$
\begin{aligned}
& \#_{m}(A, \Gamma)=\#_{m}(A)+\#_{m}(\Gamma) \\
& \#_{m}(\Lambda)=0
\end{aligned}
$$

$\overline{{ }^{1} \overline{\max }=\min ; \overline{\min }}=\max$. We leave the parameter $P \in \mathcal{P}$ implicit. 


$$
\begin{aligned}
& \frac{}{A \Rightarrow A} i d \quad \frac{\Gamma \Rightarrow A \quad \Delta(A) \Rightarrow B}{\Delta(\Gamma) \Rightarrow B} C u t \\
& \frac{\Gamma \Rightarrow A \quad \Delta(B) \Rightarrow C}{\Delta(\Gamma, A \backslash B) \Rightarrow C} \backslash L \quad \frac{A, \Delta \Rightarrow B}{\Delta \Rightarrow A \backslash B} \backslash R \\
& \frac{\Gamma \Rightarrow A \quad \Delta(B) \Rightarrow C}{\Delta(B / A, \Gamma) \Rightarrow C} / L \quad \frac{\Delta, A \Rightarrow B}{\Delta \Rightarrow B / A} / R \\
& \frac{\Delta(A, B) \Rightarrow C}{\Delta(A \bullet B) \Rightarrow C} \bullet L \quad \frac{\Delta \Rightarrow A \quad \Gamma \Rightarrow B}{\Delta, \Gamma \Rightarrow A \bullet B} \bullet R \\
& \frac{\Delta(B) \Rightarrow C}{\Delta(A \wedge B) \Rightarrow C} \wedge L 1 \quad \frac{\Delta(A) \Rightarrow C}{\Delta(A \wedge B) \Rightarrow C} \wedge L 2 \\
& \frac{\Delta \Rightarrow B \quad \Delta \Rightarrow C}{\Delta \Rightarrow B \wedge C} \wedge R \\
& \frac{\Delta(A) \Rightarrow C \quad \Delta(B) \Rightarrow C}{\Delta(A \vee B) \Rightarrow C} \vee L \\
& \frac{\Delta \Rightarrow C}{\Delta \Rightarrow B \vee C} \vee R 1 \quad \frac{\Delta \Rightarrow B}{\Delta \Rightarrow B \vee C} \vee R 2
\end{aligned}
$$

Fig. 1. Rules for the additives and multiplicatives 
And we define the counts $\#_{\min / \max }$ of a sequent by:

$$
\#_{m}(\Delta \Rightarrow A)=\#_{m}(A)-\#_{m}(\Delta)
$$

Given an arbitrary atomic type $P$ we have the following theorem:

(1) Theorem (Soundness of LA w.r.t. $\left.\#_{m, P}^{p}(A)\right)$

If $\Delta \Rightarrow A$ is an $\mathbf{L A}$ provable sequent then:

$$
\#_{\text {min }}(\Delta \Rightarrow A) \leq 0 \leq \#_{\text {max }}(\Delta \Rightarrow A)
$$

Proof. By induction on the length of Cut-free LA derivations. In the following, i.h. will abbreviate induction hypothesis.

- Axiom case. If $A=P$ then:

$$
\begin{aligned}
& \#_{\text {min }}(A \Rightarrow A) \\
& \#_{\text {min }}(A)-\#_{\text {min }}(A)=1-1=0=1-1= \\
& \#_{\text {max }}(A)-\#_{\text {max }}(A) \\
& \#_{\text {max }}(A \Rightarrow A)
\end{aligned}
$$

Otherwise, if $A$ is an atomic type $Q$ different from $P$ we have:

$$
\#_{\text {min }}(A \Rightarrow A)=0-0=\#_{\text {max }}(A \Rightarrow A)
$$

$-\wedge$ left rule:

$$
\frac{\Delta(A) \Rightarrow C}{\Delta(A \wedge B) \Rightarrow C} \wedge L
$$

We have that:

$$
\begin{array}{ll}
\#_{\text {min }}(\Delta(A \wedge B) \Rightarrow C) & = \\
\#_{\text {min }}(C)-\left(\#_{\text {min }}(A \wedge B)+\#_{\text {min }}(\Delta(\Lambda))\right) & = \\
\#_{\text {min }}(C)-\max \left(\#_{\text {min }}(A), \#_{\text {min }}(B)\right)-\#_{\text {min }}(\Delta(\Lambda)) & \leq \\
\#_{\text {min }}(C)-\#_{\text {min }}(A)-\#_{\text {min }}(\Delta(\Lambda)) & = \\
\#_{\text {min }}(\Delta(A) \Rightarrow C) & \text { i.h. }
\end{array}
$$

On the other hand:

$$
\begin{aligned}
0 \stackrel{\text { i.h. }}{\leq} & \#_{\text {max }}(\Delta(A) \Rightarrow C)= \\
& \#_{\text {max }}(C)-\#_{\text {max }}(A)-\#_{\text {max }}(\Delta(\Lambda)) \leq \\
& \left.\#_{\text {max }}(C)-\text { min }_{\text {max }}(A), \#_{\text {max }}(B)\right)-\#_{\text {max }}(\Delta(\Lambda))= \\
& \#_{\text {max }}(C)-\#_{\text {max }}(A \wedge B)-\#_{\text {max }}(\Delta(\Lambda))= \\
& \#_{\text {max }}(\Delta(A \wedge B) \Rightarrow C)
\end{aligned}
$$

$-\wedge$ right rule:

$$
\frac{\Delta \Rightarrow B \quad \Delta \Rightarrow C}{\Delta \Rightarrow B \wedge C} \wedge R
$$


Suppose that $\max \left(\#_{\min }(B), \#_{\text {min }}(C)\right)=\#_{\text {min }}(B)$. We have that,

$$
\begin{aligned}
& \#_{\text {min }}(\Delta \Rightarrow B \wedge C) \quad= \\
& \#_{\text {min }}(B \wedge C)-\#_{\text {min }}(\Delta) \quad= \\
& \max \left(\#_{\text {min }}(B), \#_{\text {min }}(C)\right)-\#_{\text {min }}(\Delta)= \\
& \#_{\text {min }}(B)-\#_{\text {min }}(\Delta) \quad= \\
& \#_{\text {min }}(\Delta \Rightarrow B) \quad \stackrel{\text { i.h. }}{\leq} 0
\end{aligned}
$$

If $\max \left(\#_{\min }(B), \#_{\text {min }}(C)\right)=\#_{\text {min }}(C)$ we get similarly:

$$
\begin{array}{ll}
\#_{\text {min }}(\Delta \Rightarrow B \wedge C) & = \\
\#_{\text {min }}(B \wedge C)-\#_{\text {min }}(\Delta) & = \\
\left.\max _{\text {min }}(B), \#_{\text {min }}(C)\right)-\#_{\text {min }}(\Delta) & = \\
\#_{\text {min }}(C)-\#_{\text {min }}(\Delta) & = \\
\#_{\text {min }}(\Delta \Rightarrow C) & \text { i.h. }
\end{array}
$$

On the other hand, suppose that $\min \left(\#_{\text {max }}(B), \#_{\text {max }}(C)\right)=\#_{\text {max }}(B)$

$$
\begin{aligned}
0 \stackrel{\text { i.h. }}{\leq} & \#_{\text {max }}(\Delta \Rightarrow B)= \\
& \#_{\text {max }}(B)-\#_{\text {max }}(\Delta)= \\
& \min \left(\#_{\text {max }}(B), \#_{\text {max }}(C)\right)-\#_{\text {max }}(\Delta)= \\
& \#_{\text {max }}(B \wedge C)-\#_{\text {max }}(\Delta)= \\
& \#_{\text {max }}(\Delta \Rightarrow B \wedge C)
\end{aligned}
$$

Similarly, if we have that $\min \left(\#_{\text {max }}(B), \#_{\text {max }}(C)\right)=\#_{\text {max }}(C)$ we obtain:

$$
0 \leq \#_{\text {max }}(\Delta \Rightarrow B \wedge C)
$$

- $\vee$ left rule:

$$
\frac{\Delta(A) \Rightarrow C \quad \Delta(B) \Rightarrow C}{\Delta(A \vee B) \Rightarrow C} \vee L
$$

Suppose that $\min \left(\#_{\text {min }}(A), \#_{\text {min }}(B)\right)=\#_{\text {min }}(A)$. We have that:

$$
\begin{aligned}
& \#_{\text {min }}(\Delta(A \vee B) \Rightarrow C) \quad= \\
& \#_{\text {min }}(C)-\#_{\text {min }}(A \vee B)-\#_{\text {min }}(\Delta(\Lambda))= \\
& \#_{\text {min }}(C)-\min \left(\#_{\text {min }}(A), \#_{\text {min }}(B)\right)-\#_{\text {min }}(\Delta(\Lambda))= \\
& \#_{\text {min }}(C)-\#_{\text {min }}(A)-\#_{\text {min }}(\Delta(\Lambda))= \\
& \#_{\text {min }}(\Delta(A) \Rightarrow C) \quad \stackrel{\text { i.h. }}{\leq} 0
\end{aligned}
$$

Similarly, if we have that $\min \left(\#_{\text {min }}(A), \#_{\text {min }}(B)\right)=\#_{\text {min }}(B)$ we obtain:

$$
\begin{array}{ll}
\#_{\text {min }}(\Delta(A \vee B) \Rightarrow C) & = \\
\#_{\text {min }}(C)-\#_{\text {min }}(A \vee B)-\#_{\text {min }}(\Delta(\Lambda)) & = \\
\left.\#_{\text {min }}(C)-\min _{\text {min }}(A), \#_{\text {min }}(B)\right)-\#_{\text {min }}(\Delta(\Lambda)) & = \\
\#_{\text {min }}(C)-\#_{\text {min }}(B)-\#_{\text {min }}(\Delta(\Lambda)) & = \\
\#_{\text {min }}(\Delta(B) \Rightarrow C) & \text { i.h. } \\
\text { a } 0
\end{array}
$$


On the other hand, if we have $\max \left(\#_{\max }(A), \#_{\max }(B)\right)=\#_{\max }(A)$ :

$$
\begin{aligned}
0 \stackrel{\text { i.h. }}{\leq} & \#_{\text {max }}(\Delta(A) \Rightarrow C)= \\
& \quad \#_{\text {max }}(C)-\#_{\text {max }}(A)-\#_{\text {max }}(\Delta(\Lambda))= \\
& \quad \#_{\text {max }}(C)-\max \left(\#_{\text {max }}(A), \#_{\text {max }}(B)\right)+\#_{\text {max }}(\Delta(\Lambda)) \\
& \quad \#_{\text {max }}(\Delta(A \vee B) \Rightarrow C)
\end{aligned}
$$

Similarly, if we have $\max \left(\#_{\max }(A), \#_{\max }(B)\right)=\#_{\max }(B)$ we get the desired result.

$-\vee$ right rule:

$$
\frac{\Delta \Rightarrow B}{\Delta \Rightarrow B \vee C} \vee R
$$

We have that:

$$
\begin{array}{ll}
\#_{\min }(\Delta \Rightarrow B \vee C) & = \\
\#_{\min }(B \vee C)-\#_{\min }(\Delta) & = \\
\min \left(\#_{\min }(B), \#_{\min }(C)\right)-\#_{\min }(\Delta) & \leq \\
\#_{\min }(B)-\#_{\min }(\Delta) & = \\
\#_{\min }(\Delta \Rightarrow B) & \text { i.h. }
\end{array}
$$

On the other hand:

$$
\begin{aligned}
& \text { i.h. }{ }_{\max }(\Delta \Rightarrow B)= \\
& \quad \#_{\max }(B)-\#_{\text {max }}(\Delta) \leq \\
& \left.\quad \max \#_{\max }(B), \#_{\max }(C)\right)-\#_{\max }(\Delta)= \\
& \quad \#_{\max }(\Delta \Rightarrow B \vee C)
\end{aligned}
$$

- / left rule:

$$
\frac{\Gamma \Rightarrow A \quad \Delta(B) \Rightarrow C}{\Delta(B / A, \Gamma) \Rightarrow C} / L
$$

We have that:

$$
\frac{\#_{\min }(\Gamma \Rightarrow A) \stackrel{\text { i.h. }}{\leq} 0 \quad \#_{\min }(\Delta(B) \Rightarrow C) \stackrel{\text { i.h. }}{\leq} 0}{\#_{\min }(C) \underbrace{-\left(\#_{\min }(B)-\#_{\min }(A)\right)}_{=-\#_{\text {min }}(B / A)}-\#_{\min }(\Gamma)-\#_{\min }(\Delta(\Lambda)) \leq 0} \text { Adding both inequations }
$$

Where the last inequation corresponds to

$$
\#_{\text {min }}(\Delta(B / A, \Gamma) \Rightarrow C) \leq 0
$$

On the other hand:

$$
\frac{\stackrel{\text { i.h. }}{\leq} \#_{\max }(\Gamma \Rightarrow A) \quad 0 \stackrel{\text { i.h. }}{\leq} \#_{\max }(\Delta(B) \Rightarrow C)}{0 \leq \#_{\max }(C)-\#_{\max }(\Delta(\Lambda))-\#_{\max }(\Gamma) \underbrace{-\left(\#_{\max }(B)-\#_{\max }(A)\right)}_{=-\#_{\max }(B / A)}} \text { Adding both inequations }
$$


Where the last inequation corresponds to:

$$
0 \leq \#_{\max }(\Delta(B / A, \Gamma) \Rightarrow C)
$$

- / right rule

$$
\frac{\Delta, A \Rightarrow B}{\Delta \Rightarrow B / A} / R
$$

We have that:

$$
\#_{\text {min }}(\Delta, A \Rightarrow B)=\underbrace{\#_{\text {min }}(B)-\#_{\text {min }}(A)}_{=\#_{\text {min }}(B / A)}-\#_{\text {min }}(\Delta) \stackrel{\text { i.h. }}{\leq} 0
$$

Where the last inequation corresponds to:

$$
\#_{\text {min }}(\Delta \Rightarrow B / A) \leq 0
$$

On the other hand:

$$
\begin{aligned}
& \stackrel{\text { i.h. }}{\leq} \#_{\text {max }}(\Delta, A \Rightarrow B)= \\
& \#_{\text {max }}(B)-\#_{\text {max }}(A)-\#_{\text {max }}(\Delta)= \\
& \#_{\text {max }}(B / A)-\#_{\text {max }}(\Delta)
\end{aligned}
$$

Where the last inequation corresponds to:

$$
0 \leq \#_{\max }(\Delta \Rightarrow B / A)
$$

- $\backslash \mathrm{L}$ rule: as $/ \mathrm{L}$

- $\backslash \mathrm{R}$ rule: as $/ \mathrm{R}$

- - left rule:

$$
\frac{\Delta(A, B) \Rightarrow C}{\Delta(A \bullet B) \Rightarrow C} \bullet L
$$

We have that:

$$
\#_{\text {min }}(C)-\#_{\text {min }}(\Delta(\Lambda))-\#_{\text {min }}(A)-\#_{\text {min }}(B) \stackrel{\text { i.h. }}{\leq} 0
$$

Where the last inequation corresponds to:

$$
\#_{\text {min }}(\Delta(A \bullet B) \Rightarrow C) \leq 0
$$

On the other hand:

$$
0 \stackrel{\text { i.h. }}{\leq} \#_{\text {max }}(C)-\#_{\text {max }}(\Delta(\Lambda))-\#_{\text {max }}(A)-\#_{\text {max }}(B)
$$

Where the last inequation corresponds to:

$$
0 \leq \#_{\max }(A(A \bullet B) \Rightarrow C)
$$


- right rule:

$$
\frac{\Delta \Rightarrow A \quad \Gamma \Rightarrow B}{\Delta, \Gamma \Rightarrow A \bullet B} \bullet R
$$

We have that:

$$
\frac{\#_{\text {min }}(A)-\#_{\text {min }}(\Delta) \stackrel{\text { i.h. }}{\leq} 0 \quad \#_{\text {min }}(B)-\#_{\text {min }}(\Gamma) \stackrel{\text { i.h. }}{\leq} 0}{\#_{\text {min }}(A \bullet B)-\#_{\text {min }}(\Delta)-\#_{\text {min }}(\Gamma) \leq 0} \text { Adding both inequations }
$$

Where the last inequation corresponds to:

$$
\#_{\text {min }}(\Delta, \Gamma \Rightarrow A \bullet B) \leq 0
$$

On the other hand:

$$
\frac{0 \stackrel{\text { i.h. }}{\leq} \#_{\max }(A)-\#_{\max }(\Delta) \quad 0 \stackrel{\text { i.h. }}{\leq} \#_{\max }(B)-\#_{\max }(\Gamma)}{0 \leq \#_{\max }(A \bullet B)-\#_{\max }(\Delta)-\#_{\max }(\Gamma)} \text { Adding both inequations }
$$

Where the last inequation corresponds to:

$$
0 \leq \#_{\max }(\Delta, \Gamma \Rightarrow A \bullet B)
$$

This completes the proof.

\subsection{Exemplification}

In this section, by way of example we give some underivable sequents which are falsified by the count check. Let $P$ and $Q$ be two atomic types:

1) $\nvdash_{\mathrm{LA}} P \Rightarrow P \wedge Q$

Consider the count check with respect to $P$. We have then that:

$$
\begin{aligned}
& \#_{\text {max }}(P \Rightarrow P \wedge Q)= \\
& \#_{\text {max }}(P \wedge Q)-1= \\
& \min (1,0)-1=-1 \nsucceq 0
\end{aligned}
$$

Therefore we falsify sequent 1 ).

2) $\nvdash_{\mathbf{L A}} P \vee Q \Rightarrow P$

Consider the count check with respect to $Q$. We have then that:

$$
\begin{array}{ll}
\#_{\text {max }}(P \vee Q \Rightarrow P) & = \\
\#_{\text {max }}(P)-\#_{\text {max }}(P \vee Q) & = \\
0-\max (0,1) & =-1 \nsucceq 0
\end{array}
$$

Therefore we falsify sequent 2 ). 
3) $\nvdash_{\mathrm{LA}} P \vee Q \Rightarrow P \wedge Q$

Consider the count check with respect to $P$. We have then that:

$$
\begin{aligned}
& \#_{\max }(P \vee Q \Rightarrow P \wedge Q)= \\
& \#_{\max }(P \wedge Q)-\#_{\max }(P \vee Q)= \\
& \min (1,0)-\max (1,0) \quad=0-1=-1 \nsupseteq 0
\end{aligned}
$$

Therefore we falsify sequent 3 ).

4) $\nvdash_{\mathbf{L A}} P \Rightarrow P \bullet P$

Consider the count check with respect to $P$. We have then that:

$$
\begin{array}{ll}
\#_{\text {min }}(P \Rightarrow P \bullet P) & = \\
\#_{\text {min }}(P \bullet P)-\#_{\text {min }}(P) & = \\
2-1 & =1 \not \leq 0
\end{array}
$$

Therefore we falsify sequent 4 ).

\section{Count invariance for bracket modalities}

In the Lambek calculus with bracket modalities (Morrill 1992[10]; Moortgat 1995[8]) configurations are bracketed; for linguistic applications see Morrill (1994[14], ch. 7) or Morrill (2011[15], ch. 5). We extend the logic LA with bracket modalities, and we denote it LAb; configurations may now include brackets. The logical rules for bracket modalities are as shown in Figure 2.

$$
\begin{array}{cc}
\frac{\Gamma(A) \Rightarrow B}{\Gamma\left(\left[[]^{-1} A\right]\right) \Rightarrow B}[]^{-1} L & \frac{[\Gamma] \Rightarrow B}{\Gamma \Rightarrow[]^{-1} B}[]^{-1} R \\
\frac{\Gamma([A]) \Rightarrow B}{\Gamma(\langle>A) \Rightarrow B}\langle\rangle L & \frac{\Gamma \Rightarrow B}{[\Gamma] \Rightarrow\langle\rangle B}\langle\rangle R
\end{array}
$$

Fig. 2. Logical rules for bracket modalities

We can define bracket counts $\#_{\min / \max ,[]}$ as follows: ${ }^{2}$

$$
\begin{array}{ll}
\#_{m}(P) & =0 \text { for } P \in \mathcal{P} \\
\#_{m}(\langle>A) & =\#_{m}(A)+1 \\
\#_{m}\left([]^{-1} A\right) & =\#_{m}(A)-1
\end{array}
$$

\footnotetext{
${ }^{2}$ We leave implicit the reference to [].
} 
The clauses for the multiplicative and additive connectives are the same as those given in the previous section. We extend the bracket count to configurations thus:

$$
\begin{aligned}
& \#_{m}(A, \Gamma)=\#_{m}(A)+\#_{m}(\Gamma) \\
& \#_{m}([\Gamma])=\#_{m}(\Gamma)+1 \\
& \#_{m}(\Lambda)=0
\end{aligned}
$$

(Naturally for an atom $P, \#_{m, P}([\Gamma])=\#_{m, P}(\Gamma)$.) Where $m \in\{\min , \max \}$, the $\min / \max -$ count of a sequent is again:

$$
\#_{m}(\Delta \Rightarrow A)=\#_{m}(A)-\#_{m}(\Delta)
$$

The soundness theorem (1) extends to bracket modalities.

Proof. Extending the proof of (1) to bracket modalities.

-\langle\rangle left rule:

$$
\frac{\Delta([A]) \Rightarrow B}{\Delta(\langle>A) \Rightarrow B}\langle\rangle L
$$

We have that for $m \in\{\min , \max \}$ :

$$
\#_{m}([A])=\#_{m}(\langle\rangle A)
$$

It follows that for $m \in\{\min , \max \}$ :

$$
\begin{aligned}
& \#_{m}(\Delta([A]) \Rightarrow B)= \\
& \#_{m}(\Delta(\langle\rangle A) \Rightarrow B)
\end{aligned}
$$

And therefore, by i.h.:

$$
\begin{aligned}
& \#_{\text {min }}(\Delta(\langle\rangle A) \Rightarrow B) \leq 0 \\
& 0 \leq \#_{\text {max }}(\Delta(\langle\rangle A) \Rightarrow B)
\end{aligned}
$$

-\langle\rangle right rule:

$$
\frac{\Delta \Rightarrow A}{[\Delta] \Rightarrow\langle\rangle B}\langle\rangle R
$$

We have that for $m \in\{\min , \max \}$ :

$$
\begin{aligned}
& \#_{m}([\Delta] \Rightarrow\langle\rangle A)=\#_{m}(\langle\rangle A)-\#_{m}([\Delta])= \\
& \left(\#_{m}(A)+1\right)-\#_{m}(\Delta)-1= \\
& \#_{m}(A)-\#_{m}(\Delta)= \\
& \#_{m}(\Delta \Rightarrow A)
\end{aligned}
$$

It follows that by i.h:

$$
\begin{aligned}
& \left.\#_{\min }([\Delta]) \Rightarrow\langle\rangle B\right) \leq 0 \\
& 0 \leq \#_{\max }([\Delta] \Rightarrow\langle\rangle B)
\end{aligned}
$$


- []$^{-1}$ left rule:

$$
\frac{\Delta(A) \Rightarrow B}{\Delta\left(\left[[]^{-1} A\right]\right) \Rightarrow B}[]^{-1} L
$$

We have that for $m \in\{\min , \max \}$ :

$$
\#_{m}\left(\left[[]^{-1} A\right]\right)=\left(\#_{m}(A)-1\right)+1=\#_{m}(A)
$$

It follows that for $m \in\{\min , \max \}$ :

$$
\#_{m}\left(\Delta\left(\left[[]^{-1} A\right]\right) \Rightarrow B\right)=\#_{m}(\Delta(A) \Rightarrow B)
$$

And therefore by i.h.:

$$
\begin{aligned}
& \#_{\min }\left(\Delta\left(\left[[]^{-1} A\right]\right) \Rightarrow B\right) \leq 0 \\
& 0 \leq \#_{\max }\left(\Delta\left(\left[[]^{-1} A\right] \Rightarrow B\right)\right.
\end{aligned}
$$

-[]$^{-1}$ right rule:

$$
\frac{[\Delta] \Rightarrow A}{\Delta \Rightarrow[]^{-1} A}[]^{-1} R
$$

We have that for $m \in\{\min , \max \}$ :

$$
\begin{aligned}
& \#_{m}([\Delta] \Rightarrow A)= \\
& \#_{m}(A)-\#_{m}(\Delta)-1= \\
& \left(\#_{m}(A)-1\right)-\#_{m}(\Delta)= \\
& \#_{m}\left(\Delta \Rightarrow[]^{-1} A\right)
\end{aligned}
$$

It follows that by i.h.:

$$
\begin{aligned}
& \#_{\min }\left(\Delta \Rightarrow[]^{-1} A\right) \leq 0 \\
& 0 \leq \#_{\max }\left(\Delta \Rightarrow[]^{-1} A\right)
\end{aligned}
$$

This completes the proof.

\subsection{Exemplification}

We consider some examples of underivable sequents which are falsified by the count invariant extended to bracket modalities. Let $N$ and $S$ be two atomic types.

1) $\nvdash_{\text {LAb }} N,(\langle\rangle N) \backslash S \Rightarrow S$

We have that the following count check with respect to []:

$$
\begin{aligned}
\#_{\text {min }}(N,(\langle\rangle N) \backslash S \Rightarrow S) & =\#_{\text {min }}(S)-\#_{\text {min }}(N)-\#_{\text {min }}((\langle\rangle N) \backslash S) \\
& =\#_{\text {min }}(S)-\#_{\text {min }}(N)-\#_{\text {min }}(S)+\#_{\text {min }}(\langle\rangle N) \\
& =\#_{\text {min }}(S)-\#_{\text {min }}(N)-\#_{\text {min }}(S)+\left(\#_{\text {min }}(N)+1\right) \\
& =0-0-0+(0+1)=1 \not 0
\end{aligned}
$$

Therefore we falsify sequent 1 ). 
2) $\nvdash_{\text {LAb }}[[N]],(\langle\rangle N) \backslash S \Rightarrow S$

Consider the count check with repect to []:

$$
\begin{aligned}
\#_{\max }([[N]],(\langle\rangle N) \backslash S \Rightarrow S) & =\#_{\max }(S)-\#_{\max }([[N]])-\#_{\max }((\langle\rangle N) \backslash S) \\
& =0-(1+1+0)-(0-(1+0)) \\
& =-2+1=-1 \nsucceq 0
\end{aligned}
$$

Therefore we falsify sequent 2).

3) $\nvdash_{\mathbf{L A b}}\left[S,\left(S \backslash\left([]^{-1}[]^{-1} S\right)\right) / S\right] \Rightarrow S$

Consider the count check with repect to []:

$$
\begin{aligned}
\#_{\text {min }}\left(\left[S,\left(S \backslash\left([]^{-1}[]^{-1} S\right)\right) / S\right] \Rightarrow S\right) & =\#_{\text {min }}(S)-\left(1+\#_{\text {min }}(S)+\left(\#_{\text {min }}(S)-2\right)-2 \cdot \#_{\text {min }}(S)\right) \\
& =0-(1-2)=1 \not \leq 0
\end{aligned}
$$

Therefore we falsify sequent 3 ).

\section{Conclusion: discriminatory power}

Our proposal for count invariance comprises two inequations. These are parameterised by atoms or brackets. If we assume that the likelyhood of satisfying one arbitrary inequation by chance is $1 / 2$, the likelyhood of satisfying one inequation for $n$ atoms or brackets is $1 / 2^{n}$. But if there are two inequations, as in our case, the chance of satisfying the two is $1 / 2 \times 1 / 2=1 / 4$, and the probability of satisfying the two equations for $n$ atoms or brackets is $1 / 4^{n}$. Thus the discriminatory capacities of one or both of our count invariants together grow with the number of atoms as follows:

\begin{tabular}{l|ll}
$n$ & $2^{n}$ & $4^{n}$ \\
\hline 1 & 2 & 4 \\
2 & 4 & 16 \\
3 & 8 & 64 \\
4 & 16 & 256 \\
5 & 32 & 1024 \\
6 & 64 & 4096 \\
7 & 128 & 16384 \\
8 & 256 & 65536
\end{tabular}

Clearly the count invariant is sound for multiplicative-additive linear logic since it is a criterion sensitive to occurences and in no way depends on commutativity or non-commutativity. In the same way it extends immediately to the deterministic connectives of the (dis)placement calculus of Morrill, Valentín and Fadda (2011[13]) since these form residuated families like the Lambek connectives. Furthermore we think it is possible to extend it to the nondeterministic discontinuous connectives since these are defined using additives. Finally, we have begun experimenting with implementation of the new count invariant in the context of the categorial parser/theorem-prover CatLog (Morrill 2012[12]). 


\section{Appendix}

Another count invariant could be defined using mutual recursion with respect to polarities (of types) and $m=\max$ or $\min$. Where polarity $p=\bullet$ or $\circ$ represents antecedent (input) and succedent (output) respectively and $m=\max$ or min, we define by mutual recursion as follows counts $\#_{\max / \min , P}$ on types, leaving the parameter $P \in \mathcal{P}$ implicit: $^{3}$

$$
\begin{aligned}
& \#_{m}^{p}(P)=1 \\
& \#_{m}^{p}(Q)=0 \text { for } Q \in \mathcal{P}-\{P\} \\
& \#_{m}^{p}(A \bullet B)=\#_{m}^{p}(A)+\#_{m}^{p}(B) \\
& \#_{m}^{p}(A \backslash C)=\#_{m}^{p}(C)-\#_{\bar{m}}^{p}(A) \\
& \#_{m}^{p}(C / B)=\#_{m}^{p}(C)-\#_{\bar{m}}^{p}(B) \\
& \#_{m}^{\circ}(A \wedge B)=m\left(\#_{m}^{\circ}(A), \#_{m}^{\circ}(B)\right) \\
& \#_{m}^{\circ}(A \wedge B)=\bar{m}\left(\#_{m}^{\circ}(A), \#_{m}^{\circ}(B)\right) \\
& \#_{m}^{\circ}(A \vee B)=\bar{m}\left(\#_{m}^{\circ}(A), \#_{m}^{\circ}(B)\right) \\
& \#_{m}^{\circ}(A \vee B)=m\left(\#_{m}^{\circ}(A), \#_{m}^{\circ}(B)\right)
\end{aligned}
$$

We extend the counts $\#_{\min / \max }^{\bullet}$ to configurations by the following:

$$
\begin{aligned}
& \#_{m}^{\bullet}(A, \Gamma)=\#_{m}^{\bullet}(A)+\#_{m}^{\bullet}(\Gamma) \\
& \#_{m}^{\bullet}(\Lambda)=0
\end{aligned}
$$

And we define the counts $\#_{\min / \max }$ of a sequent by:

$$
\#_{m}(\Delta \Rightarrow A)=\#_{\bar{m}}^{\bullet}(\Delta)-\#_{m}^{\circ}(A)
$$

\section{(2) Lemma}

The following equality holds:

$$
\#_{\bar{m}}^{\bullet}(A)=\#_{m}^{\circ}(A)(\star)
$$

Proof. By induction on the complexity of LA types (as usual, i.h. abbreviates induction hypothesis):

- Atomic case: obvious.

- Product case: obvious (using i.h.).

- Slashes. Consider / ( is completely similar):

$$
\begin{aligned}
& \#_{\bar{m}}^{\bullet}(C / A)=\#_{\bar{m}}^{\bullet}(C)-\#_{m}^{\circ}(A) \\
& \#_{m}^{\circ}(C / A)=\#_{m}^{\circ}(C)-\#_{\bar{m}}^{\bullet}(A)
\end{aligned}
$$

By i.h. $\#_{\bar{m}}^{\bullet}(C)=\#_{m}^{\circ}(C)$ and $\#_{m}^{\circ}(A)=\#_{\bar{m}}^{\bullet}(A)$, whence $\#_{\bar{m}}^{\bullet}(C / A)=\#_{m}^{\circ}(C / A)$.

- Conjunction:

$$
\begin{aligned}
& \#_{\bar{m}}^{\bullet}(A \wedge B)=m\left(\#_{\bar{m}}^{\bullet}(A), \#_{\bar{m}}^{\bullet}(B)\right) \\
& \#_{m}^{o}(A \wedge B)=m\left(\#_{m}^{\circ}(A), \#_{m}^{\circ}(B)\right)
\end{aligned}
$$

By i.h. $\#_{\bar{m}}^{\bullet}(A)=\#_{m}^{\circ}(A)$ and $\#_{m}^{\circ}(B)=\#_{\bar{m}}^{\bullet}(B)$, whence $\#_{\bar{m}}^{\bullet}(A \wedge B)=\#_{m}^{\circ}(A \wedge B)$.

\footnotetext{
${ }^{3} \bar{\bullet}=0 ; \bar{o}=\bullet ; \overline{\max }=\min ; \overline{\min }=\max$.
} 
- Disjunction: similar to the case of conjunction.

This count invariant satisfies also the soundness theorem (1). By using the previous lemma (2) we can almost mimick the proof from (1). The definition of count invariant we present in this appendix has turned out to be interesting for an ongoing research on a count invariant extended to the exponential modality of linear logic ! (Girard 1987[2]).

\section{References}

1. J. M. Andreoli. Logic programming with focusing in linear logic. Journal of Logic and Computation, 2(3):297-347, 1992.

2. Jean-Yves Girard. Linear logic. Theoretical Computer Science, 50:1-102, 1987.

3. H. Hendriks. Studied flexibility. Categories and types in syntax and semantics. PhD thesis, Universiteit van Amsterdam, ILLC, Amsterdam, 1993.

4. Mark Hepple. Normal form theorem proving for the Lambek calculus. In H. Karlgren, editor, Proceedings of COLING, Stockholm, 1990.

5. M. Kanazawa. The Lambek calculus enriched with additional connectives. Journal of Logic, Language and Information, 1:141-171, 1992.

6. E. König. Parsing as natural deduction. In Proceedings of the Annual Meeting of the Association for Computational Linguistics, Vancouver, 1989.

7. J. Lambek. On the Calculus of Syntactic Types. In Roman Jakobson, editor, Structure of Language and its Mathematical Aspects, Proceedings of the Symposia in Applied Mathematics XII, pages 166-178. American Mathematical Society, Providence, Rhode Island, 1961.

8. Michael Moortgat. Multimodal linguistic inference. Journal of Logic, Language and Information, 5(3, 4):349-385, 1996. Also in Bulletin of the IGPL, 3(2,3):371-401, 1995.

9. G. Morrill. Grammar and Logical Types. In Martin Stockhof and Leen Torenvliet, editors, Proceedings of the Seventh Amsterdam Colloquium, pages 429-450, 1990. Also in G. Barry and G. Morrill, editors, Studies in Categorial Grammar, Edinburgh Working Papers in Cognitive Science, Volume 5, pages 127-148: 1990. Revised version published as Grammar and Logic, Theoria, LXII, 3:260-293, 1996.

10. Glyn Morrill. Categorial Formalisation of Relativisation: Pied Piping, Islands, and Extraction Sites. Technical Report LSI-92-23-R, Departament de Llenguatges i Sistemes Informàtics, Universitat Politècnica de Catalunya, 1992.

11. Glyn Morrill. Logic Programming of the Displacement Calculus. In Sylvain Pogodalla and Jean-Philippe Prost, editors, Proceedings of Logical Aspects of Computational Linguistics 2011, LACL'11, Montpellier, number LNAI 6736 in Springer Lecture Notes in AI, pages 175-189, Berlin, 2011. Springer.

12. Glyn Morrill. CatLog: A Categorial Parser/Theorem-Prover. In LACL 2012 System Demonstrations, Logical Aspects of Computational Linguistics 2012, pages 13-16, Nantes, 2012.

13. Glyn Morrill, Oriol Valentín, and Mario Fadda. The Displacement Calculus. Journal of Logic, Language and Information, 20(1):1-48, 2011. Doi 10.1007/s10849-010-9129-2.

14. Glyn V. Morrill. Type Logical Grammar: Categorial Logic of Signs. Kluwer Academic Publishers, Dordrecht, 1994.

15. Glyn V. Morrill. Categorial Grammar: Logical Syntax, Semantics, and Processing. Oxford University Press, New York and Oxford, 2011.

16. J. van Benthem. Language in Action: Categories, Lambdas, and Dynamic Logic. Number 130 in Studies in Logic and the Foundations of Mathematics. North-Holland, Amsterdam, 1991. Revised student edition printed in 1995 by the MIT Press. 